\title{
Sensitivity and specificity of optic disc variables and analysis of a new variable (MP/D) for glaucoma diagnosis with the Glaucoma-Scope
}

Yves Lachkar, Howard Cohn

\begin{abstract}
Aim-In an attempt to use the quantitative optic disc measurements of the Glaucoma-Scope (OIS Sacramento, CA, USA) to distinguish glaucomatous from normal optic discs, a new variable was investigated, the mean disc corrected for the disc size by dividing by the disc area: MP/D. Methods-Glaucoma-Scope disc evaluation was performed on 81 eyes of 51 patients split into the following groups based on Humphrey 24-2 visual field and clinical criteria of glaucoma: chronic glaucoma $n=27$ (including only early, $n=17$, and low tension glaucoma, $n=10$ ), ocular hypertension $n=24$, pseudoglaucomatous large discs, $n=12$, and normal eyes, $n=18$. Classic optic disc variables (the vertical and horizontal c/d ratios, and the c/d area) were compared with the new $M P / D$ index calculating receiver operating characteristic curves.

Results-The MP/D ratio was able to identify the glaucomatous eyes more easily than other ratios. Areas under the curves were: 0.91 (MP/D); 0.87 (c/d area); 0.85 (c/d vertical); and 0.80 (c/d horizontal). The MP/D index was also correlated with the mean deviation $(r=0.466 ; p=0.001)$. Conclusion-MP/D may prove useful in detecting glaucomatous optic nerve damage and could be an interesting screening tool for primary open angle glaucoma. (Br f Ophthalmol 1997;81:846-851)
\end{abstract}

Various functional tests and morphological examinations are used to assist the clinician in the diagnosis of glaucoma and follow up of patients. Automated static threshold perimetry represents one of the best standard diagnostic tests. Optic disc examination is currently performed but the clinical examination of the optic disc is subjective and interpretations are often inconsistent even among expert observers. $^{1-3}$ Recently, automated instruments have been developed for quantitative analysis of the optic nerve head to provide early information on the presence of structural glaucomatous damage.

In an attempt to use the quantitative optic disc measurements of the Glaucoma-Scope (OIS, Sacramento, CA, USA) to help distinguish glaucomatous from normal optic discs, we investigated a new variable, the mean disc depth corrected for the disc size by dividing by the disc area: MP/D. Despite the different nature of the morphological data obtained with the Glaucoma-Scope and the functional data obtained with the automated static perimetry correlation was expected.

\section{Material and methods}

The Glaucoma-Scope consists of an optical head mounted on a slit lamp assembly. The optical head includes a raster pattern projection system and a video camera to capture images. Data are saved immediately on a hard disk and later archived to an optical laser disk where they can be easily accessed. The minimum necessary pupillary diameter following pharmacological mydriasis in the study was $4.0 \mathrm{~mm}$.

Approximately 25 parallel horizontal dark and light pairs are projected at an angle of $9^{\circ}$ to the optic nerve head $(\mathrm{ONH})$ using near infrared light $(750 \mathrm{~nm})$. As the lines pass over the surface of the $\mathrm{ONH}$ they are deflected proportional to the depth of the surface. A video image records these deflections and computer algorithms translate them into depth numbers. There are 26 edges of the 25 lines projected onto the optic nerve and each edge has 350 real data points. Therefore, there are approximately 9100 real data points per image. The reference surface for the depth measurements is defined by linear interpolation of data falling in two vertical $50 \mu \mathrm{m}$ columns placed $350 \mu \mathrm{m}$ nasal and temporal to the disc margin.

For the patient's first examination, the operator selects a reference point for future image registration and outlines the disc margin (eight points) and major vessels to provide landmarks on the printout. The disc border is defined as the inner margin of Elschnig's ring. The margin and vessel drawings do not affect data calculation. Computer algorithms perform a best fit analysis on data in an area subtending $4^{\circ}$ surrounding the reference point to register the current and baseline images. A single operator obtained three images from each eye. The operator may control image focus, line focus, and illumination independently. The computer selected a single image for processing based on the quality of focus and line contrast. The topographic measurements were performed by the same operator (YL).

Results are presented in a grey scale (Fig 1). Numeric values are represented on the printout; each cell represents the average of an area $69 \times 138 \mathrm{~mm}$ in size. On subsequent visits, depth measurements are automatically compared with measurements made at the initial
Accepted for publication 26 June 1997 


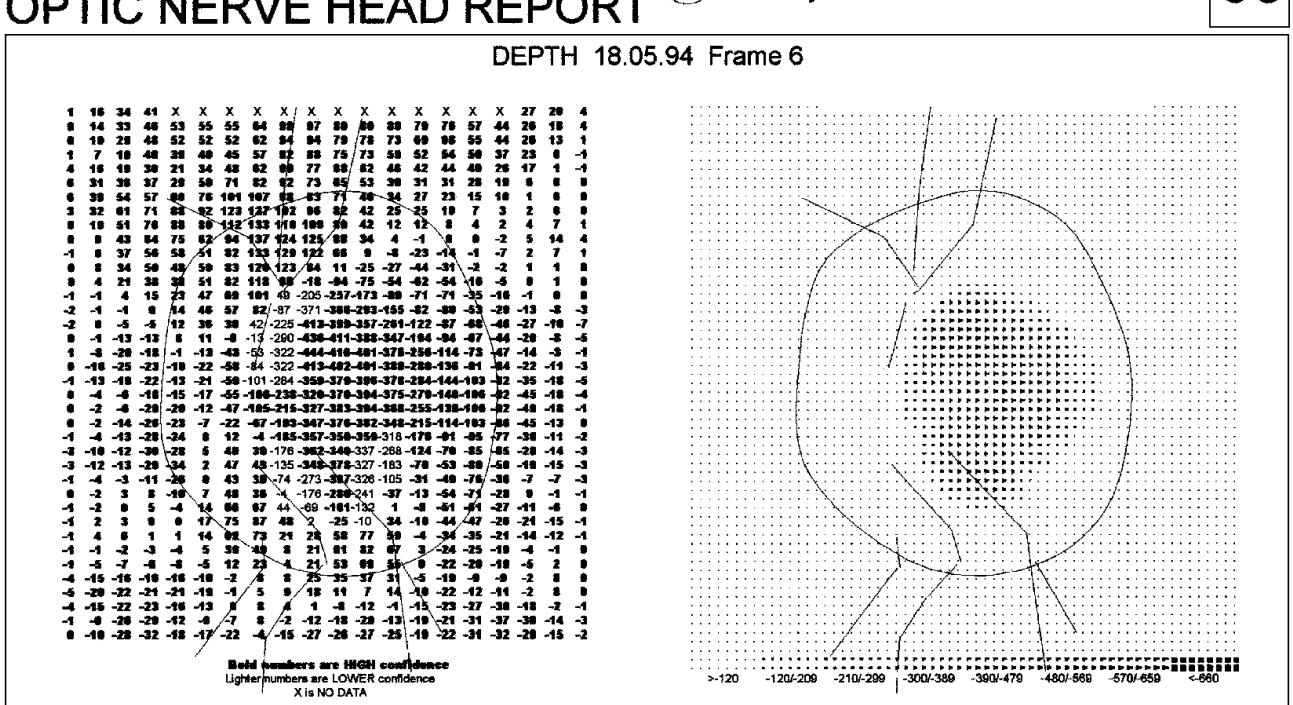

Figure 1 Glaucoma-Scope line discrimination analysis. Numbers are in $\mu m$.

visit, and a map is generated showing any change in depth values (of $<50 \mu \mathrm{m}$ : threshold of the test).

Version 3.12 also presents a three dimensional image (Fig 2) with the calculation of different ratios and measurements: vertical cup/ disc (c/d) ratio, horizontal c/d ratio, c/d area ratio, cup area $\left(\mathrm{mm}^{2}\right)$. The mean position of the disc (MP disc) is also calculated corresponding to the mean height value for all data points within the disc margin relative to the zero reference plane (average of all depth measurements within the disc). The MP total region is the mean position of the total region (average of all depth measurements on the topography map). The software uses the disc tracing drawn by the operator as the disc edge. The cup is calculated by the instrument from the position of all points that are estimated to be $120 \mu \mathrm{m}$ or more below the reference. The cup edge is drawn at the $120 \mu \mathrm{m}$ depth boundary inside the disc. The rim is defined as the area remaining between the edge of the cup and the outline of the disc edge. The disc diameter is taken as the longest vertical line that can be drawn through the disc margin. In the present study version 3.12 was used and corrections were made by the computer for refraction and for the quality of the images. The real size is calculated using the formula devised by Bengston and Krakau. ${ }^{4}$ This formula corrects the disc measurements according to an estimate of the magnification of the eye, based on the refraction.

The mean disc depth corrected for the disc size by dividing by the disc area (MP/D) was also investigated and compared with the others variables usually given.

We examined 81 eyes of 51 patients with chronic glaucoma $(n=27)$, including early glaucoma $(\mathrm{n}=17)$, low tension glaucoma $(n=10)$, ocular hypertension $(n=24)$, pseudoglaucomatous large discs $(n=12)$, and normal eyes $(n=18)$ with program $24-2$ of the Humphrey perimeter and with the OIS GlaucomaScope.

Chronic glaucoma was defined as intraocular pressure more than $22 \mathrm{~mm} \mathrm{Hg}$ with the following visual field defects ${ }^{5}$ :

(1) early glaucoma

- the mean deviation (MD) less than -6 $\mathrm{dB}$

- a pattern deviation plot: fewer than $25 \%$ of the points depressed below the 5\% level and fewer than 10 points depressed below the $1 \%$ level

- no point in the central $5^{\circ}$ with a sensitivity of less than $15 \mathrm{~dB}$

(2) moderately advanced glaucoma:

- the MD less than $-12 \mathrm{~dB}$

- a pattern plot: fewer than $50 \%$ of the points are depressed below the 5\% level and fewer than 20 points below the $1 \%$ level

- no point in the central $5^{\circ}$ with a sensitivity of $0 \mathrm{~dB}$

- only one hemifield may have a point with sensitivity of $<15 \mathrm{~dB}$ within $5^{\circ}$ of fixation.

Low tension glaucoma was defined as an intraocular pressure without therapy less than $22 \mathrm{~mm} \mathrm{Hg}$ with early or moderately advanced visual field damage and a clinical c/d ratio more than 0.6. Patients with ocular hypertension had intraocular pressure greater than $22 \mathrm{~mm} \mathrm{Hg}$ without visual field damage and a clinical $\mathrm{c} / \mathrm{d}$ ratio not greater than 0.6 . Patient with a $\mathrm{c} / \mathrm{d}$ ratio larger than 0.7 without visual field damage and with intraocular pressures below $22 \mathrm{~mm} \mathrm{Hg}$ were classified as having a pseudoglaucomatous disc. Normal eyes had normal IOP, optic disc, and visual field (24-2 Humphrey). All patients had previous visual field examinations with the Humphrey 24-2 program. For comparison of the difference between different groups we used the analysis of 


\section{Ophthalmic Imaging Systems Glaucoma-Scope OPTIC NERVE HEAD REPORT PAGE 2}

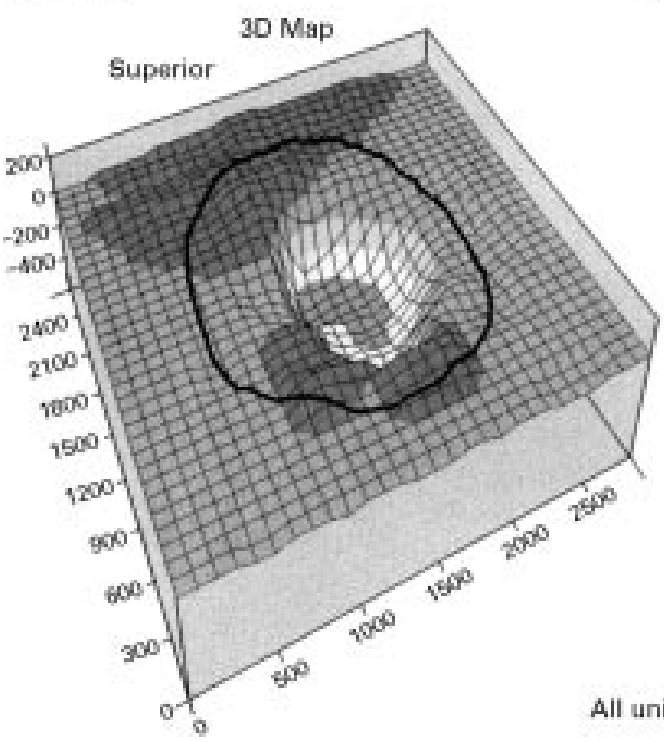

$-480-360-240-120$

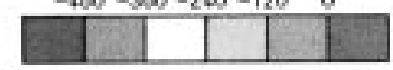

\begin{tabular}{|l|l|l|}
\cline { 2 - 3 } \multicolumn{1}{c|}{} & \multicolumn{1}{c|}{ CURRENT } & PREVIOUS \\
\hline Date & 18.05 .94 & \\
\hline Analysis & 2 & \\
\hline Frame & 6 & \\
\hline CiD ratio vert & 0.58 & \\
\hline CiD ratio horiz & 0.55 & \\
\hline Cupioise area ratio & 0.30 & \\
\hline Max disc diam vert & 1.914 & \\
\hline Max disc diam horiz & 1.787 & \\
\hline Disc area (mm ${ }^{2}$ ) & 2.875 & \\
\hline Cup area imm ${ }^{2}$ ) & 0.848 & \\
\hline MP dise & -87.3 & \\
\hline MP total region & -28.0 & \\
\hline Fx sphere & +02.00 & \\
\hline Rx cylinder & $-00.75 \times 072$ & \\
\hline
\end{tabular}

Name:

ID Code: LAC080735

Physician: Dr Yves Lachkar

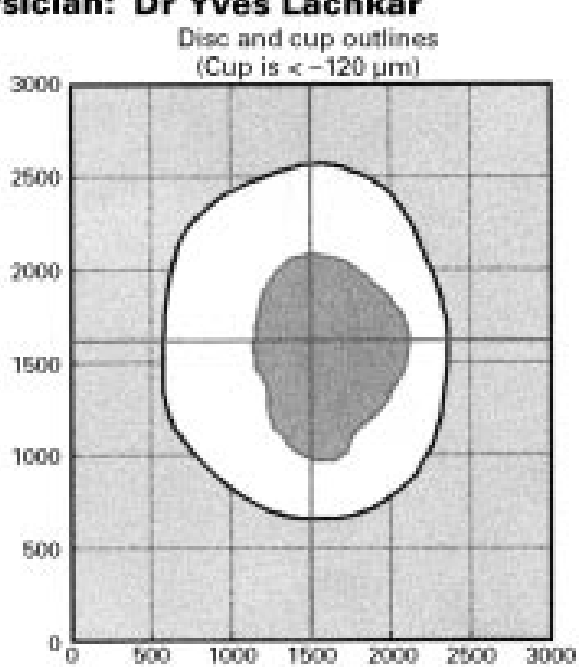

All units are in $\mu \mathrm{m}$
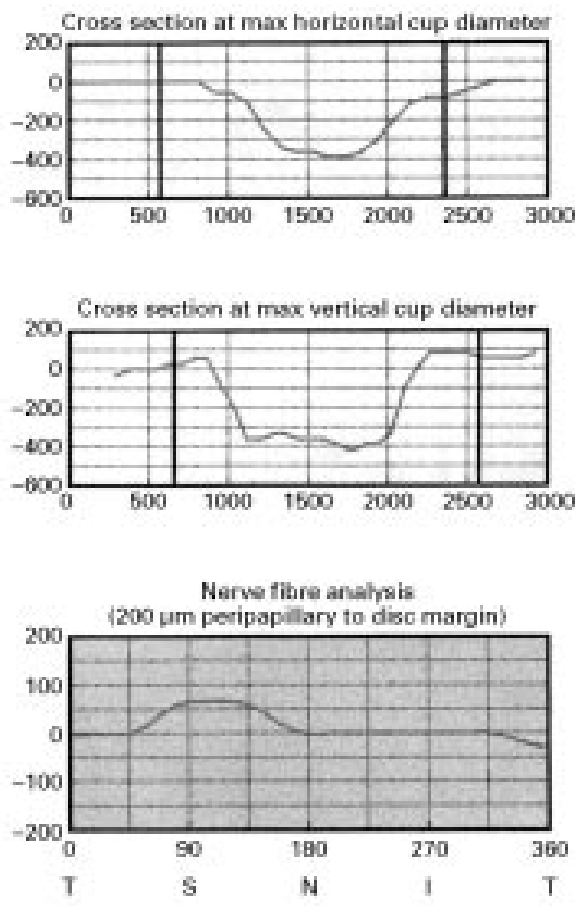

Figure 2 Glaucoma-Scope analysis, version 3.12 (three dimensional image). Calculation of different ratios: vertical cupldisc (c/d) ratio, horizontal cld ratio, cld area ratio, cup area $\left(\mathrm{mm}^{2}\right)$. The mean position of the disc (MP disc) corresponds to the mean height value for all data points within the disc margin relative to the zero reference plane (average of all depth measurements within the disc). The MP total region is the mean position of the total region (average of all depth measurements on the topography map).

variance (one way ANOVA). Age and refraction are not statistically different in the normal group, the ocular hypertension group, and the group with early or moderately advanced glaucoma $(\mathrm{p}>0.2)$.

\section{Results}

Table 1 lists the patient characteristics.

In the current study the ROC (receiver operating characteristic) curves for the stand- ard disc variables and for the MP/D were obtained. Areas under the curves were: 0.91 (MP/D); 0.87 (c/d area); 0.85 (c/d vertical); and 0.80 (c/d horizontal) (Fig 3). Reviewing the different disc variables and their separation characteristics, the MP/D ratio showed the best separation power. Different values are presented in Figure 4. The MP/D index was also correlated with the mean deviation $(r=0.465$; $\mathrm{p}=0.001)($ Fig 5). 
Table 1 Patient characteristics

\begin{tabular}{llcccc}
\hline & Group 1 Normal & Group 2 GLD & Group 3 OHT & Group 4 LTG \\
\hline Eyes (n) & 18 & 12 & 24 & & 10 \\
Age (mean (range)) & $52.77(35-67)$ & $63.66(43-82)$ & 57.5 & $(34-67)$ & $66.16(46-79)$ \\
Refraction (mean (range)) & $0.75(-1.75 /+2.00)$ & $1.00(-2.00 /+2.25)$ & $1.25(-1.75 /+2.75)$ & $-0.75(-3.25 /+2.25)$ & $-1.28(-4.25 /+3.25)$ \\
MD (Humphrey) (mean (SD)) & $-0.26(1.55)$ & $-1.26(1.03)$ & $-0.693(1.65)$ & $-9.9(7.55)$ & $-4.32(2.33)$ \\
N deficits p<0.5 (mean (SD)) & 0 & $0.63(1.02)$ & 0 & $-25(11.13)$ & $-6.22(3.47)$ \\
MP/D (mean (SD)) & $20.42(15.71)$ & $-54.55(26.70)$ & $-26.34(26.74)$ & $-67.34(33.32)$
\end{tabular}

There was a statistical difference $(\mathrm{p}<0.001$; $\mathrm{F}=43.74)$ for the $\mathrm{MP} / \mathrm{D}$ ratio between the three groups who had a normal visual field (1, 2 , and 3) and group 4 (early glaucoma).

Between the normal and the ocular hypertension group the difference was not statistically different $(\mathrm{p}=0.9104 ; \mathrm{F}=0.0129)$.

Using Delong's method ${ }^{6}$ to compare areas under the ROC curves we found a highly significant difference $(t=21.9 ; \mathrm{p}<0.001)$ between the $M P / D$ variable and the other variables.

\section{Discussion}

During the past few years there have been a number of studies on the performance of traditional glaucoma screening tests. These include tonometry, clinical examination, photography of the nerve fibre layer, three dimensional imaging of the optic nerve, and a number of subjective tests. Elevated IOP is an important risk factor for glaucoma, but the actual sensitivity of IOP evaluation as a single test would appear to lie at just half the $100 \%{ }^{7}$ Electrophysiological and psychophysical tests of vision are interesting tools to separate glaucomatous from normal eyes but are subject to fluctuations and sometimes are too long.

Even among acknowledged glaucoma experts there is wide variation in the subjective assessment of optic nerve status. ${ }^{1}$ The traditional cut off value of 0.5 or 0.6 (cup/disc ratio) has a sensitivity below $50 \%$, and lower cut off levels seriously compromise specificity. In the Baltimore Survey ${ }^{8}$ the sensitivity and specificity of vertical cup to disc ratio in detecting primary open angle glaucoma were respectively $52 \%$ and $89 \%$ for a cup/disc ratio at 0.5 and $29 \%$ and $98 \%$ for a cup to disc more than or equal to 0.6 . The size of the disc is as important a factor in the interpretation of discs as the cup to disc ratio. The size can be calculated clinically ${ }^{9}{ }^{10}$ but this variable has not been routinely evaluated for mass screening.

Nerve fibre layer evaluation has also been evaluated with good sensitivity and specifi-

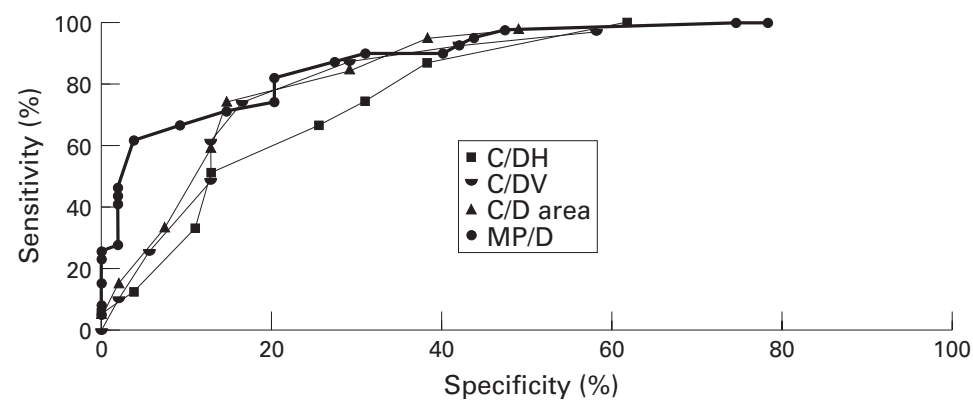

Figure 3 Receiver operating characteristic curves for the standard disc variables and for the $M P / D$.

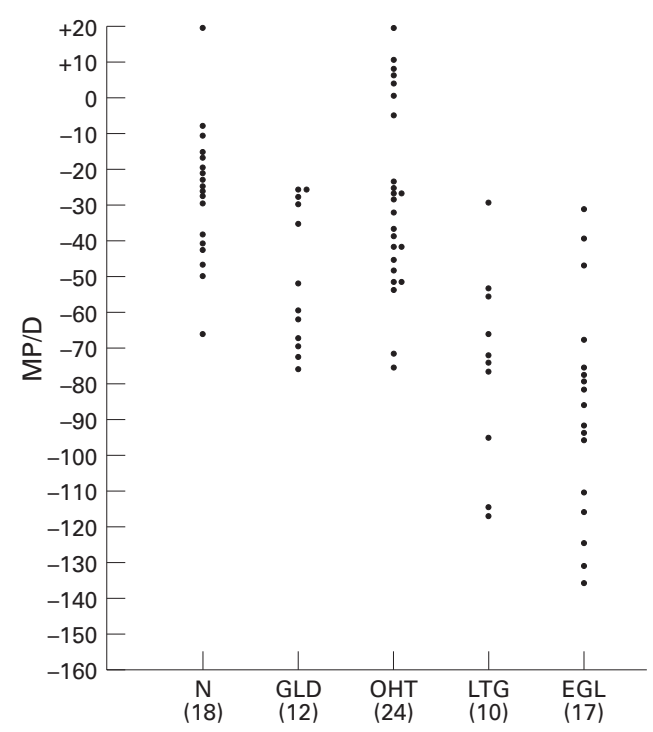

Figure $4 M P / D$. Mean disc depth corrected for the disc size by dividing the disc area. $N=$ normal; $O H T=o c u l a r$ hypertension; $G L D=$ glaucoma-like disc

(pseudoglaucomatous discs); $L T G=$ low tension glaucoma; EGL=early glaucoma.

city. ${ }^{11}{ }^{12}$ However, media opacities focusing problems and poor quality photographs can results in a large number of photographs. As pointed out by Sommer, ${ }^{12}$ photographic techniques are not standardised and it is difficult to obtain high quality photographs of the nerve fibre layer in older patients.

Computer assisted optical imaging of the optic nerve could make mass screening a real possibility because it offers objective assessment, ease of use, speed, and the ability to obtain good quality images in eyes with media opacities.

The Glaucoma-Scope is one of these analysers. Previous studies ${ }^{13-15}$ have shown good reproducibility of the optic nerve head topo-

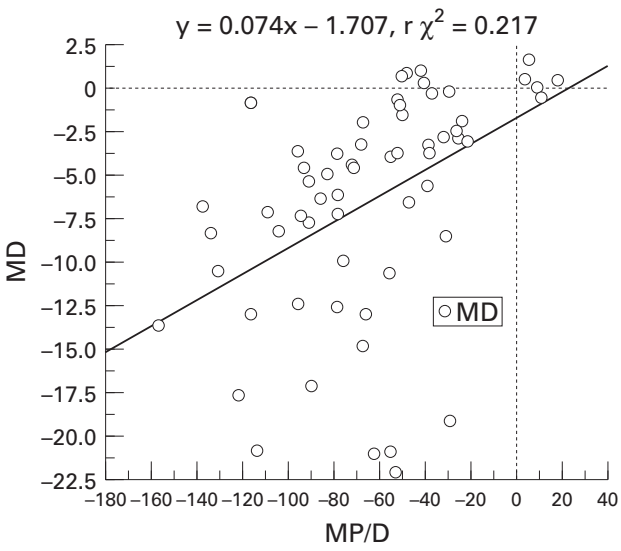

Figure 5 Correlation between MP/D (Glaucoma-Scope) and MD (Humphrey). 
graphic measurements with the GlaucomaScope. However the potential of the 3.09 version as a diagnostic tool in glaucoma may be limited, ${ }^{16}$ because the quality of the image is not corrected by the computer and because this version gives only the grey scale image (Fig 1)

In this study we deliberately chose only patients with early or moderately advanced visual field loss (low tension glaucoma), because if patients with advanced visual field loss had been included, differentiation of disc change would inevitably have been much better, with higher values of $\mathrm{MP} / \mathrm{D}$, but of much less academic and clinical interest. According to previous studies, ${ }^{17}$ the horizontal $\mathrm{c} / \mathrm{d}$ ratio was less able to discriminate between groups than the vertical c/d ratio. The MP/D variable provided the best separation of patient groups and we focused our analysis on this variable. With a cut off value of -55 we obtained a sensitivity of $82 \%$ and a specificity of $79.2 \%$, with this single variable.

In an experimental model in monkey eyes using the Glaucoma-Scope, Quigley and Pease $^{18}$ showed that the loss of nerve fibre induced by elevated intraocular pressure was more closely related to change in the mean position of the disc calculated with the Glaucoma-Scope than to change in cup/disc ratio. Our clinical approach, which included the size of the disc, showed that the best variable for glaucoma diagnosis is probably not the cup/disc ratio.

Caprioli has shown that a combination of variables can detect glaucomatous disc damage, but it is still not certain what variables or combination of them are the best indicators of glaucomatous damage. ${ }^{19}{ }^{20}$ In another study he had also shown that the best variable for glaucoma diagnosis is the analysis of the nerve fibre layer. ${ }^{21}$ This analysis can also be obtained with the Glaucoma-Scope and could be combined with the MP/D index to improve the sensibility and specificity of the optic nerve analysis. Gundersen et $a l^{22}$ using the same device (Glaucoma-Scope), calculated the narrowest rim width within $60^{\circ}$ and $90^{\circ}$ sectors centred across the vertical diameter of the optic nerve and compared these variables with those usually given by the Glaucoma-Scope (cup area, rim area, horizontal and vertical cup/disc ratio). Using ROC curves, minimum rim width $60^{\circ}$ and $90^{\circ}$ showed the best separating characteristics. These variables were measured manually using a ruler on the size corrected disc and cup outline chart and global indices used for comparison were not corrected for the disc size. Many optic disc variables vary physiologically with optic disc size and correction for this variability may improve separation of glaucoma patients from normals. However, selection of subjects on the basis of optic disc morphology introduces a bias in this type of study.

The Glaucoma-Scope makes a correction for ocular magnification based on refraction only. ${ }^{4}$ The dimension of the eye that has the greatest effect on ocular magnification is the axial length. ${ }^{43}$ It has been shown that for emmetropic eyes with the same corneal curva- ture $(97.7 \mathrm{~mm})$ the axial length by ultrasound may vary between 22.0 and $25.2 \mathrm{~mm} .^{24}$ Thus, the correction made by the Glaucoma-Scope is only an approximation.

The ability of computer assisted optic nerve analysis to detect early glaucomatous visual field damage has also previously been studied by Mikelberg et $a l^{25}$ with the Heidelberg retina tomograph (HRT). The data were analysed using the reference plane of the software 1.10 using a method incorporating the height of the papillomacular bundle as reference level. Fourteen variables have been extracted from the ONH topography image, but without the mean cup depth. This variable is also calculable with the HRT. With the standard reference level, setting at the standard value of $320 \mu \mathrm{m}$ below the surface, the important variables found were the third moment and the maximum cup depth. With the papillomacular bundle reference level, the volume above the reference level was important, and with age correction, height variation in contour replaced maximum depth in the analysis. There is a great variability in the appearance of the normal optic nerve, and so it is difficult to have a single variable of the $\mathrm{ONH}$ shape that will allow determination of glaucoma. Nevertheless, the $\mathrm{MP} / \mathrm{D}$ variable adds important information to disc analysis and seems to be an interesting one to include in the analysis of the $\mathrm{ONH}$.

The authors have no proprietary interest in the equipment described here.

Presented at the ARVO meeting, Fort Lauderdale, Florida, May 1995.

We wish to thank Mr Andrew MacNaught (Western Eye Hospital London) for his contribution to the manuscript and Mrs Jane Wadsworth (Imperial College School of Medicine at St Mary's Hospital) for her statistical advice.

1 Lichter PR. Variability of expert observers in evaluating the optic disc. Trans Am Ophthalmol Soc 1976;74:532-72.

2 Tielsch JM, Katz J, Quigley HA, Miller NR, Sommer A.

Intraobserver and interobserver agreement in measurement of optic disc characteristics. Ophthalmology 1988;95: ment of

3 Varma R, Steinman WC, Scott IU. Expert agreement in evaluating the optic disc for glaucoma. Ophthalmology 1992;99:215-21.

4 Bengston B, Krakau CET. Correction of optic disc measurements on fundus photographs. Graefes Arch Clin Exp Ophthalmol 1991;230:24-8.

5 Hoddap E, Parrish RK, Anderson DR. Clinical decision in glaucoma. St Louis: CV Mosby, 1993:52-61.

6 DeLong ER, DeLong DM, Clarke-Pearson DL. Comparing the areas under two or more correlated receiver operating characteristic curves: a nonparametric approach. Biometrics 1998;44:837-45.

7 Sponsel WE. Tonometry in question: can visual screening tests play a more decisive role in glaucoma diagnosis an management. Surv Ophthalmol 1989;33(suppl):291-300.

8 Tielsch JM, Katz J, Singh O, Quigley HA, Gottsch JD, Javitt , Sommer A. A population based evaluation of glaucoma screening: the

9 Ruben S. Estimation of optic disc size using indirect biomiRuben S. Estimation of optic disc size using
croscopy. Br F Ophthalmol 1994;78:363-4.

10 Jonas J, Papastathopoulos K. Ophthalmic measurement of the optic disc. Ophthalmology 1995;102:1102-6.

11 Sommer A, Quigley HA, Robin AL, Miller NR, Katz J, Arkell S. Evaluation of nerve fiber layer assesment. Arch Ophthalmol 1984;102:1766-71.

12 Sommer A. Retinal nerve fiber layer. Am $\mathcal{f}$ Ophthalmol 1995;120:665-7.

13 Hoskins HD, Hetherington J, Glenday M, Samuels S, Verdooner S. Repeatability of the Glaucoma-Scope measurements of the optic nerve head topography. F Glaucoma 1994;4:17-27.

14 Hamzavi S, Stewart WC, Jackson GJ, Thompson TL. Reproducibility of the Glaucoma-Scope in cadaver eyes. Acta Ophthalmol Scand 1995;73:264-7.

15 Pendergast SD, Shields B. Reproducibility of optic nerve head topographic measurements with the OIS Glaucomahead topographic measurements with

16 Dan JA, Beleyea DA, Lieberman MF, Stamper RL. Evaluation of optic disc measurements with the Glaucoma-Scope. f Glaucoma 1996;5:1-8. 
17 Damms T, Danheim F. Sensitivity and specificity of optic disc parameters in chronic glaucoma. Invest Ophthalmol Vis

18 Quigley H, Pease M. Change in the optic disc and nerve fiber layer estimated with the Glaucoma-Scope in monkey eyes. F Glaucoma 1996;5:106-16.

19 Caprioli J. Discrimination between normal and glaucomatous eyes. Invest Ophthalmol Vis Sci 1992;33:153-9.

20 O'Connor DJ, Zeyen T, Caprioli J. Comparison of methods to detect glaucomatous optic nerve damage. Ophthalmology 1993;100:1498-503.

21 Caprioli J. The contour of the juxtapapillary nerve fiber layer in glaucoma. Ophthalmology 1990;97:358-66.
22 Gundersen K, Heijl A, Bengtson B. Sensitivity and specificity of structural parameters in chronic glaucoma. Acta Ophthalmol Scand 1996;74:120-

23 Bennet AG, Rudnicka AR, Edgar DF. Improvements on Littman's method of determining the size of retinal features by fundus photography. Graefes Arch Clin Exp Ophthalmol 1994;232:361-7.

$24 \mathrm{Gernet} \mathrm{H}$. Ein beitrag zur frage der emmetropisation. Ophthalmologica 1964;147:235-43.

25 Mikelberg FS, Parfitt CM, Swindale NV, Graham SL, Drance SM, Gosine R. Ability of the Heidelberg Retina Tomograph to detect early glaucomatous visual field loss. $\mathcal{F}$ Glaucoma 1995;4:242-7. 